\title{
Institucionalização na velhice: uma revisão sistemática da literatura sobre preditores em contexto de Estruturas Residenciais para Pessoas Idosas (ERPI) Institutionalization in old age: a systematic review of literature on predictors in the context of Long-term Care (LTC)
}

\author{
Ricardo Crispim \\ (D) https://orcid.org/0000-0002-8355-1830 \\ Universidade de Coimbra, Portugal \\ rmscrispim@hotmail.com
}

Recibido: 30-09-2021

Aceptado: 23-10-2021

\begin{abstract}
Resumen
Um vasto conjunto de pessoas idosas necessita de cuidados específicos. A ausência de rede de suporte e/ou a existência deficitária desta impulsiona a solicitar apoio às ERPI. O objetivo deste trabalho é investigar, à luz da literatura nacional e internacional, os fatores preditores da institucionalização em ERPI. Trata-se de uma revisão sistemática da literatura, realizada nas bases de dados WEB OF SCIENCE, ScienceDirect, SCOPUS e demais artigos relevantes. A amostra final foi constituída por 26 estudos. Os preditores de institucionalização de pessoas idosas identificados com forte evidência foram: perda de saúde e autonomia das pessoas mais velhas, bem como da inexistência, incapacidade e ausência de rede de apoio informal que garanta o apoio nas AVD e AIVD daqueles que experienciam a velhice. Estudos desta natureza oferecem alertas acerca dos públicos que atualmente recorrem às ERPI e aqueles que futuramente surgirão. Fornecem-nos indicadores valiosos que permitirão (re)configurar os cuidados às pessoas idosas nos estabelecimentos residenciais agora e no futuro. Por fim, permite aos interventores sociais (re)fundarem matérias essenciais de atuação e reflexão na Gerontologia e, no caso particular, no Serviço Social.
\end{abstract}

Palavras-chave: Estruturas Residenciais para Pessoas Idosas (ERPI), fatores preditivos; pessoas idosas, vivência da velhice, revisão sistemática de literatura.

\begin{abstract}
A wide range of elderly people need specific care. The lack of a support network and/or its deficient existence drives them to request support in an IEP. The purpose of this article is to investigate, in the light of the national and international literature, the predictors of institutionalization in LTC. is a systematic literature review, conducted in the WEB OF SCIENCE, ScienceDirect, SCOPUS databases and other relevant articles. The final sample consisted of 26 studies. The predictors of institutionalization of older people identified with strong evidence were: loss of health and autonomy of older people, as well as the inexistence, disability and absence of informal support network that ensures support in the ADLs and IADLs of those experiencing old age. Studies of this nature provide warnings about the people who currently use IORPs and those who will use them in the future. They provide us with valuable indicators that will allow us to (re)configure the care of elderly people in residential facilities now and in the future. Finally, it allows social interventionists to (re)establish essential subjects for action and reflection in Gerontology and, in this particular case, in Social Work.
\end{abstract}

Keywords: Long-term Care (LTC), predictive factors, old people, old age experience, systematic literature review.

\section{Resumo}

1. Introdução|2. Metodologia | 3. Resultados | 3.1. Perdas na saúde e autonomia | 3.2. Rede de apoio informal |4. Discussão | 5. Conclusão | Referências bibliográficas

\section{Como citar este artigo}

Crispim, R. (2021): "Institucionalização na velhice: uma revisão sistemática da literatura sobre preditores em contexto de Estruturas Residenciais para Pessoas Idosas (ERPI)", methaodos. revista de ciencias sociales, 9 (2): 258-271. http://dx.doi.org/10.17502/mrcs.v9i2.499 


\section{Introdução}

Dados recentes indicam-nos que em Portugal existem mais de dois milhões de pessoas com 65 ou mais anos (PORDATA, 2021). Este facto parece acompanhar a tendência mundial, colocando Portugal como o quarto país mais envelhecido do mundo (Rosa, 2020). Isto é, com o aumento do número de pessoas idosas, o fenómeno do envelhecimento em Portugal não é mais uma realidade invisível. Razão que leva, nomeadamente no final do século XX e início do século XXI, à atenção, por exemplo, da academia e dos decisores políticos, acerca das problemáticas que envolvem a fase mais tardia da vida.

Atualmente sabe-se que o prolongamento da vida se tornou uma realidade em Portugal (Instituto Nacional de Estatística [INE], 2020) devido, em parte, ao declínio perigoso da fecundidade e ao aumento da longevidade e da esperança média de vida quer à nascença quer aos 65 anos (Cabral, 2017; Mendes, 2017). Acresce que o número de pessoas muito idosas (i.e., pessoas com mais de 85 anos) tem aumentado significativamente (Brandão et al., 2017), devido, entre outros, à diminuição das taxas de mortalidade nomeadamente em idades avançadas (Cabral, 2017; Mendes, 2017), projetando a velhice como a idade do futuro.

Em Portugal a esperança média de vida à nascença foi estimada, no ano de 2019, em 81,1 anos, mais elevada para as mulheres (83,7 anos) do que para os homens (78,1 anos) (PORDATA, 2021a). Já a esperança média de vida aos 65 anos, no ano de 2019, equivalia a 19,7 anos, sendo respetivamente de 17,8 anos e de 21,1 anos para os homens e para as mulheres com a mesma idade (PORDATA, 2021b). Estes dados sugeremnos uma pergunta: Viver mais anos é ou não acompanhado de um aumento de tempo vivido em boa saúde? Os dados parecem indicar-nos que não (PORDATA, 2020). Se tivermos em linha de consideração as limitações devido a problemas de saúde, a estimativa de anos de vida saudável à nascença, em 2018, era de 58,6 anos, mais baixa para as mulheres ( 57,5 anos) do que para os homens (59,8 anos) (PORDATA, 2020). Ao nível das doenças crónicas ou problemas de saúde prolongados, a PORDATA (2021c) esclarece a evolução ascendente deste indicador. Podemos identificar, por exemplo, que as doenças crónicas ou problemas de saúde que duram ou que podem vir a durar pelo menos seis meses assumem percentagens mais elevadas junto das mulheres (46,3\%) do que dos homens (39,6\%). A prevalência da ausência de saúde ocorre, segundo a mesma fonte, em $73,8 \%$ das pessoas idosas. Naquilo que tem de ver com limitações devido a problemas de saúde é registada uma redução de anos, isto é, 7,3 anos para a população com 65 ou mais anos, 8,2 anos para os homens e 6,9 para as mulheres (PORDATA, 2020). Na substância, os dados evidenciam uma feminização da velhice decorrente da maior esperança média de vida das mulheres, embora o acréscimo em anos se traduza numa diminuição das condições de saúde.

Observa-se que tal cenário - o aumento da esperança média de vida à nascença e aos 65 anos associada à dependência e à vulnerabilidade ocorridas predominantemente na fase mais tardia da vida acrescentam desafios à sociedade em geral e à forma como se organizam os sistemas familiares e políticos em particular.

Neste sentido, carece de se atender que à medida que as populações envelhecem, nomeadamente nas sociedades desenvolvidas e/ou em desenvolvimento, a necessidade por cuidados formais de longa duração para pessoas idosas tende a aumentar no setor privado e público (Gabinete de Planeamento e Estratégia [GPE], 2021; Rodrigues, 2017).

Atualmente, a necessidade de cuidados de longa duração parece estar associada à fase mais tardia da vida, desde logo porque a conceção biomédica do envelhecimento aliada à noção de tradicional de velhice (i.e., associada ao processo de senescência, à deterioração física, às patologias, à perda de faculdades, à inadequação, ao empobrecimento, à ineficácia, à senilidade e à tomada a cargo) tende a permanecer na maioria dos países desenvolvidos o que faz aumentar a pressão exercida junto das famílias (Rodrigues, 2017). Por tal razão, especta-se que o número de pessoas idosas com necessidade de cuidados formais aumente de um modo acentuado nas próximas décadas (Hajek et al., 2015), fenómeno este que impacta a maneira como o espaço doméstico organiza o ato cuidativo. Progressivamente, tais necessidades impulsionam as famílias a solicitar cuidados formais de longa duração junto de entidades promotoras de apoio à pessoa idosa (Comissão Europeia [CE], 2021; Ferreira da Silva et al., 2020). 
Neste sentido, o número de Estruturas Residenciais para Pessoas Idosas (ERPI) em Portugal tendem a se proliferar gradualmente devido a eventos múltiplos ${ }^{1}$ embora, em parte, se deva à atual estrutura familiar e à entrada da mulher no mercado de trabalho (Pimentel, 2012). Este facto traz à cena cuidativa dificuldades acrescidas ao papel das famílias enquanto principais instituições sociais de prestação de cuidados às pessoas idosas. Circunstâncias, desta natureza fazem aumentar a procura das famílias pelas respostas sociais formais, nomeadamente as ERPI (Lopes et al., 2018). Por isso o aumento da procura de ajuda e apoios institucionais e a emergência de um vasto setor privado ganham um novo espaço na sociedade.

A este propósito, como nos informa a Carta Social relativa a $2019^{2}$, importa referir que as respostas sociais dirigidas às pessoas idosas e/ou em situação de dependência representavam em Portugal mais de $42 \%$ do total de respostas sociais por população-alvo (Gabinete de Planeamento e Estratégia [GPE], 2021). No mesmo relatório evidencia-se que foi a conjugação das principais tipologias de respostas sociais dedicadas às pessoas idosas (i.e., ERPI, Serviço de Apoio Domiciliário e Centro de Dia) aquelas que mais cresceram em Portugal, com uma percentagem de 52\%. Os dados trabalhados neste report indicam-nos que entre o ano de 2000 e 2019, estas respostas ascenderam a 160\%, traduzindo-se num aumento superior a 2700 novas respostas num total de 7306 em 2019 (Gabinete de Planeamento e Estratégia [GPE], 2021). No caso particular das ERPI, foram efetivamente aquelas que mais cresceram no período compreendido entre 2000-2019, atingindo uma percentagem record de 173\% (Gabinete de Planeamento e Estratégia [GPE], 2021). Em termos concretos, os dados mais recentes publicados acerca desta matéria de que se conhece informam-nos que existem em Portugal 2526 ERPI nas quais vivem 99234 pessoas e onde trabalham 60 mil profissionais (SNS, 2020).

Em regimes institucionais e como nos sugere a Carta Social mais atual (Gabinete de Planeamento e Estratégia [GPE], 2021), a distribuição etária indica-nos que $86 \%$ do total das pessoas idosas que usufruem de cuidados prestados em respostas de ERPI tem 75 ou mais anos de idade. Ainda acerca da caracterização dos residentes em ERPI, destacamos o facto de em termos de capacidade para a realização de atividades básicas de vida diária, os dados indicam-nos níveis elevados de dependência dos cuidados formais prestados pelas equipas das ERPI em todas as atividades avaliadas, por contraste, por exemplo, ao Serviço de Apoio Domiciliário e ao Centro de Dia, nas quais a maioria das pessoas idosas são independentes na generalidade das atividades.

Embora a necessidade e a oferta de serviços em ERPI seja uma realidade muito presente em Portugal, dados indicam que as pessoas idosas mesmo necessitando de cuidados formais de longo prazo preferem manter-se no seu meio natural o maior tempo possível, ficando em sua casa e receber cuidados domiciliários (Pinzón-Pulido et al., 2016). Não obstante, a admissão em ERPI torna-se onerosa, tanto em termos de finanças públicas como nos sistemas familiares (Rodrigues, 2017). Além disso, associada à institucionalização ainda existirem constrangimentos vários (i.e., cuidados sociais pouco flexíveis baseados numa dinâmica paliativa concentrada na tarefa e na manutenção da saúde e da segurança) inviabilizadores da participação que tendem a diminuir a margem de independência das pessoas idosas, reduzir a autonomia e a capacidade de tomada de decisão, o autoconceito e autoestima, a privacidade, o sentido de pertença e o bem-estar, fazendo progredir as pessoas idosas numa escalada de apatia e passividade (De Medeiros et al., 2020; Nogueira et al., 2016).

Diante do exposto, embora existam estudos internacionais capazes de mapear os preditores de institucionalização de pessoas idosas em ERPI, na literatura portuguesa parece ainda não ter sida realizada qualquer revisão sistemática que nos permita conhecer e analisar os determinantes que levam as pessoas idosas a viverem numa ERPI (i.e., ao que conseguimos apurar até à data da redação deste estudo). Assim, este trabalho objetiva realizar um exercício de revisão sistemática da literatura assente em estudos nacionais e internacionais publicados com vista a analisar a previsão de institucionalização de pessoas idosas com os seguintes objetivos: (a) identificar potenciais preditores de institucionalização, e (b) sintetizar resultados mostrando evidências acerca das categorias relativas aos preditores com evidência forte.

\footnotetext{
${ }^{1}$ Alguns destes eventos são: a redução da extensão da família e o surgimento de novos modelos familiares, a redução da área das habitações, o rápido processo de urbanização e a desertificação das zonas ruais, os elevados níveis de cultura e escolarização das populações (Pimentel, 2012).

${ }^{2}$ Relatório mais recente publicado.
} 


\section{Metodologia}

Este estudo constitui uma revisão sistemática da literatura a respeito dos fatores que predizem a institucionalização de pessoas idosas em contextos de ERPI. A realização deste estudo beneficiou das seguintes diretrizes: (a) formular uma questão de investigação; (b) produzir um protocolo de investigação; (c) definir os critérios de inclusão e por conseguinte de exclusão; (d) desenvolver uma estratégia de pesquisa e pesquisar a literatura (i.e., encontrar os estudos); (e) seleção dos estudos; (f) avaliação da qualidade dos estudos; (g) extração dos dados; e (h) síntese dos dados e avaliação da qualidade da evidência. Com base no exposto, para identificação do problema desenhou-se a seguinte questão norteadora: Quais os fatores preditores da institucionalização de pessoas idosas em contextos de ERPI apresentados na literatura científica nacional e internacional?

Para a revisão sistemática da literatura utilizaram-se as seguintes bases de dados: Web of Science, Scopus, Science Direct e MEDLINE, bem como bibliografias de artigos e dissertações identificadas ${ }^{3}$. Com vista ao levantamento de produções sobre o tema de pesquisa foram utilizadas as seguintes palavras-chave, aplicadas isoladamente ou em conjunto: 'preditores' (predictors), 'institucionalização' (institutionalization), 'institucionalização em ERPI' (institutionalization in NH or $L T C^{4}$ ), 'cuidados de longa duração' (long termcare) e 'pessoas idosas' (elderly people).

Para atender ao propósito os critérios de inclusão que foram tidos em consideração são:

- trabalhos elaborados nos últimos 10 anos;

- trabalhos redigidos nos idiomas em inglês, espanhol e português (de Portugal e do Brasil);

- incluir artigos que se encontravam disponíveis na íntegra;

- $\quad$ artigos em que as população-alvo fosse a população a experienciar a velhice;

- artigos baseados em contextos de ERPI.

- $\quad$ artigos baseados em preditores de admissão de pessoas idosas em ERPI;

Após a busca nas bases de dados suprarreferidas, nos termos mencionados, foram identificados 57 estudos. Após uma leitura criteriosa das produções científicas selecionadas, foram excluídos 31 trabalhos por não atenderem aos critérios de inclusão. Considerando isso, a amostra final da literatura investigada constituiu-se por 26 artigos e/ou trabalhos (Figura 1).

Figura 1. Fluxograma que permite mostrar o processo de triagem do estudo

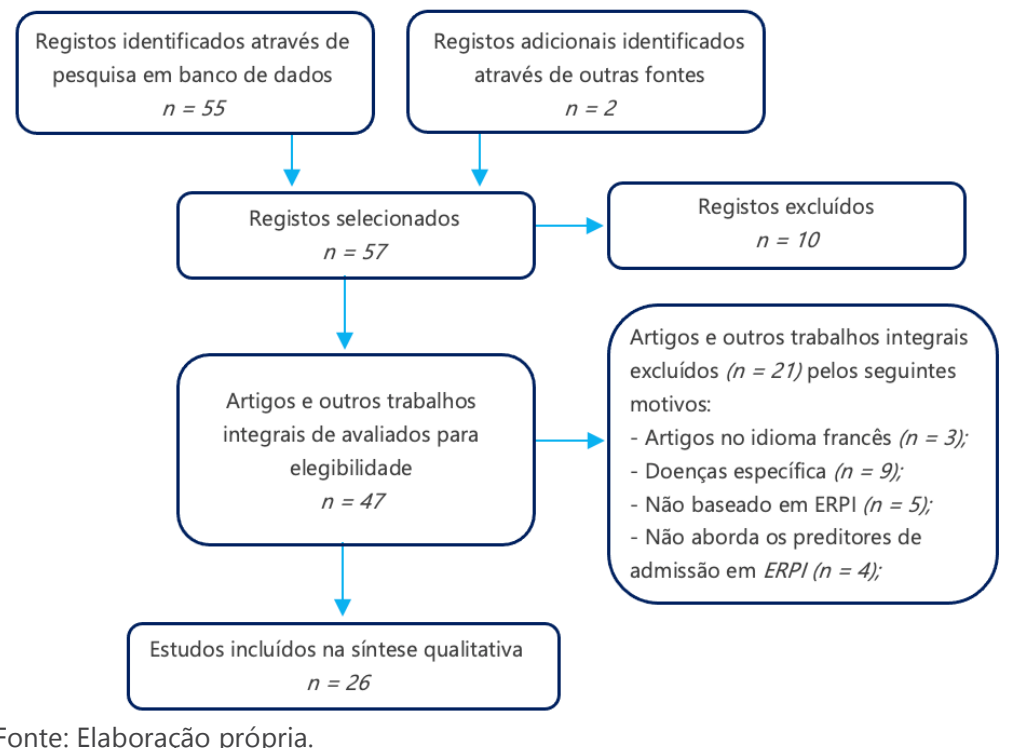

${ }^{3} \mathrm{O}$ alargamento das bases de dados prende-se com o facto de se considerar pertinente incluir trabalhos portugueses e brasileiros que não constavam na Web of Science, Scopus, Science Direct e MEDLINE acerca das matérias a trabalhar.

${ }^{4}$ Serviram de descritores em inglês as seguintes terminologias: Residential long-term care; Nursing home; Residential care; Long term care; Institutional care; Housing for the elderly; Long term care home. 


\section{Resultados}

No que se refere aos dados de publicações dos trabalhos e artigos investigados (Tabela 1), prevalecem aqueles publicados em áreas da saúde (46\%), realizados no ano de 2012 e 2017 (20\% respetivamente), no idioma inglês (61\%), publicados na Scopus (31\%), com a abordagem metodológica quantitativa (85\%). Quanto ao país de publicação, prevaleceu os Estados Unidos da América (40\%), seguido por Portugal, Inglaterra e Brasil com quatro publicações cada, Espanha com duas publicações e o Irão com uma publicação.

Tabela 1. Distribuição segundo fonte, ano de publicação, idioma, base de dados e tipo de estudo

\begin{tabular}{|c|c|c|}
\hline & $n$ & $\%$ \\
\hline \multicolumn{3}{|l|}{ Fonte } \\
\hline Publicações em áreas da saúde & 12 & 46 \\
\hline Publicações em Gerontologia e Geriatria & 9 & 35 \\
\hline Dissertações de Mestrado & 2 & 11 \\
\hline Publicações em outras áreas científicas (e.g., Psicologia, SS) & 3 & 8 \\
\hline \multicolumn{3}{|l|}{ Ano } \\
\hline 2010 & 1 & 4 \\
\hline 2011 & 3 & 11 \\
\hline 2012 & 5 & 19 \\
\hline 2013 & 1 & 4 \\
\hline 2014 & 1 & 4 \\
\hline 2015 & 2 & 8 \\
\hline 2016 & 2 & 8 \\
\hline 2017 & 5 & 19 \\
\hline 2018 & 3 & 11 \\
\hline 2019 & 1 & 4 \\
\hline 2020 & 2 & 8 \\
\hline \multicolumn{3}{|l|}{ Idioma } \\
\hline Português (de Portugal ou do Brasil) & 9 & 35 \\
\hline Inglês & 16 & 61 \\
\hline Espanhol & 1 & 4 \\
\hline \multicolumn{3}{|l|}{ Base de dados } \\
\hline Scopus & 7 & 31 \\
\hline Web of Science & 6 & 23 \\
\hline MEDLINE & 4 & 15 \\
\hline Science Direct & 2 & 8 \\
\hline SciELO & 2 & 8 \\
\hline Outros & 4 & 15 \\
\hline \multicolumn{3}{|l|}{ Tipo de estudo } \\
\hline Quantitativo & 22 & 85 \\
\hline Qualitativo & 4 & 15 \\
\hline Total & 26 & 100 \\
\hline
\end{tabular}

Fonte: Elaboração própria.

No processo de análise dos dados foram sintetizados tematicamente em categorias relativas aos preditores com evidência forte, moderada, fraca e inconclusiva da institucionalização de pessoas idosas em ERPI (Tabela 2).

Os achados para preditores com forte evidência mostram que a institucionalização de pessoas idosas em ERPI se deve fundamentalmente a três aspetos: (a) à existência de problemas psicopatológicos, neuropsiquiátricos e neuropsicológicos; (b) à ausência de cuidador informal; e (c) dependência de cuidados 
nas Atividades da Vida Diária (AVD) ${ }^{5}$ e Atividades Instrumentais da Vida Diária (AIVD) ${ }^{6}$. Parece existir uma sólida associação entre o comprometimento cognitivo e funcional e a limitação nas AVD e AIVD.

Tabela 2. Resultados sintetizados: preditores com evidência forte, moderada e fraca da institucionalização de pessoas idosas em ERPI

\begin{tabular}{|c|c|c|c|c|c|}
\hline & \\
\hline & $\begin{array}{l}\text { Evidência } \\
\text { forte }\end{array}$ & $\begin{array}{l}\text { Evidência } \\
\text { moderada }\end{array}$ & $\begin{array}{l}\text { Evidência } \\
\text { fraca }\end{array}$ & $\begin{array}{l}\text { Evidência } \\
\text { inconclusiva }\end{array}$ & $\begin{array}{c}\% \text { de } \\
\text { ocorrência }\end{array}$ \\
\hline $\begin{array}{l}\text { Problemas psicopatológicos, neuropsiquiátricos e } \\
\text { neuropsicológicos }\end{array}$ & & & & & 77 \\
\hline $\begin{array}{l}\text { Ausência de cuidador: indisponibilidade de familiares } \\
\text { para cuidar, fragilidade ou ausência de rede de apoio }\end{array}$ & & & & & 73 \\
\hline Dependência de cuidados nas AVD e AIVD & & & & & 42 \\
\hline Solidão: morar sozinho & & & & & 27 \\
\hline Idade avançada & & & & & 27 \\
\hline Presença de doenças crónicas/comorbidades várias & & & & & 31 \\
\hline Dificuldade na locomoção & & & & & 23 \\
\hline Perda de autonomia funcional & & & & & 23 \\
\hline Decisão das pessoas idosas em ingressar em ERPI & & & & & 23 \\
\hline $\begin{array}{l}\text { Moradia: contexto domiciliário inadequado, não ter } \\
\text { casa }\end{array}$ & & & & & 19 \\
\hline Género: feminino & & & & & 19 \\
\hline Conflitos familiares/abandono/violência & & & & & 15 \\
\hline Quedas & & & & & 15 \\
\hline Sobrecarga do cuidador & & & & & 15 \\
\hline Problemas emocionais & & & & & 12 \\
\hline Cancro & & & & & 12 \\
\hline Estado civil: viuvez & & & & & 12 \\
\hline Problemas financeiros/ baixos rendimentos & & & & & 12 \\
\hline Visão prejudicada & & & & & 8 \\
\hline AVC & & & & & 8 \\
\hline Fratura na anca & & & & & 8 \\
\hline Diabetes & & & & & 8 \\
\hline Doença pulmonar obstrutiva crónica & & & & & 8 \\
\hline Perda de peso não intencional & & & & & 8 \\
\hline Doença aguda & & & & & 4 \\
\hline Baixa literacia & & & & & 4 \\
\hline Audição prejudicada & & & & & 4 \\
\hline Deficiência física & & & & & 4 \\
\hline Decorrente de hospitalização & & & & & 4 \\
\hline Género: masculino & & & & & 4 \\
\hline Incontinência & & & & & 4 \\
\hline Nível de atividade baixo & & & & & 4 \\
\hline Parkinson & & & & & 4 \\
\hline Exaustão auto-relatada/baixa energia & & & & & 4 \\
\hline Violência doméstica & & & & & 4 \\
\hline
\end{tabular}

Fonte: Elaboração própria.

\footnotetext{
${ }^{5}$ Atividades da Vida Diária (AVD), são essencialmente atividades relacionadas com os seguintes itens, como por exemplo: autocuidado; mobilidade; alimentação; higiene pessoal (banho, idas à casa de banho, controle de esfíncteres); e vestir, despir, calçar.

${ }^{6}$ Atividades Instrumentais da Vida Diária (AIVD), dizem respeito a atividades que permitem a integração de uma pessoa na determinada comunidade, gerir a sua casa e a sua vida. Por exemplo: Ir às compras; gerir o dinheiro; utilizar o telefone; limpar; cozinhar; utilizar transportes.
} 


\subsection{Perdas na saúde e autonomía}

A informação acerca dos problemas psicopatológicos, neuropsiquiátricos e neuropsicológicos enquanto preditores de ingresso em ERPI foi analisada em $77 \%$ dos estudos usados neste trabalho.

Embora a esperança média de vida tenha aumentado e melhorado, o risco de doença parece aumentar com o avançar da idade (PORDATA, 2020). Neste sentido parece existir, em regra, uma propensão para que as pessoas idosas se tornem mais frágeis e dependentes de cuidados de longa duração, devido, em regra, ao processo natural de senescência presente, sobremaneira, na fase mais tardia da vida (Comissão Europeia [CE], 2021).

Observa-se, de forma bastante consistente, que a demência e/ou o diagnóstico de Alzheimer em pessoas com 65 ou mais anos se tem assumido um fator determinante para o ingresso em ERPI. A este propósito, segundo o relatório Health at a Glance 2019: OECD Indicators (2019), estima-se que em 2050 existirão nos países da OECD quase 41 milhões de pessoas com demência. Dos 36 países que compõem a OECD, em quatro destes - Japão, Itália, Portugal e Espanha -, mais de uma em cada 25 pessoas viverão com demência.

De forma muito particular, a organização não governamental Alzheimer Europe no relatório Dementia in Europe Yearbook 2019 (2019), ao analisar os dados populacionais de Portugal, referiu que no ano de 2018 existiam 183.362 pessoas com demência. A mesma fonte estima que em 2025 subirá para 220.775 o número de pessoas com demência e em 2050 para 346.905. Segundo este relatório, Portugal ultrapassará a tendência europeia, tendendo a duplicar até 2050 o número de pessoas com demência. Um fator chave nesta mudança parece ter a ver com o significativo aumento do número de pessoas longevas (Brandão et al., 2017; PORDATA, 2019; Rosa, 2020), colocando uma pressão acrescida por um lado às famílias (Pimentel e Albuquerque, 2010) e por outro lado às ERPI (Lippi Bruni e Ugolini, 2016).

No estudo de James Goodwin e sua equipa (2011), tornou-se claro que pessoas idosas com diagnóstico de demência tinham seis vezes mais possibilidade de ingresso em ERPI do que pessoas não portadoras da doença, embora no estudo inaugural e que serviu de inspiração a estudos posteriores de Melanie Luppa e a sua equipa (2009), sejam avançados dados mais agravados, atribuindo às pessoas com demência o aumento de 17 vezes mais de hipótese de ingressar em ERPI. Na verdade, este trabalho de revisão sistemática da literatura permite-nos reforçar a ideia de que a existência de quadros demenciais em pessoas idosas tende a aumentar a necessidade de institucionalização em contextos residenciais.

Decorrente do diagnóstico de demência e/ou outras doenças crónicas, tende a ocorrer nas pessoas idosas uma diminuição das capacidades em viver de forma independente e funcional. Neste trabalho investigativo, 42\% dos estudos analisados mostram-nos que a diminuição das funções das pessoas idosas (i.e., as AVD e as AIVD) com demência e/ou outras doenças crónicas beneficiam de um risco considerável no ingresso em ERPI.

Neste seguimento, outros aspetos que emergiram nos dados analisados, embora com evidência inconclusiva, tem a ver com a existência de doença aguda e a consequente hospitalização enquanto determinante de ingresso em ERPI. Estes factos, segundo Goodwin et al. (2011) e Luppa et al. (2009) podem vir acompanhadas de declínio funcional, dependência física e necessidade de cuidados prolongados, por exemplo, aquando da ocorrência de acidente vascular cerebral (AVC).

\subsection{Rede de apoio informal}

De acordo com os dados encontrados nesta investigação, ressalta-se a ausência de cuidador como um dos determinantes com evidência forte para a institucionalização em ERPI. Corroborando com este facto, a revisão integrativa da literatura realizada por Ferreira da Silva et al. (2020) e o estudo de mestrado de Pinto (2013) concluíram que a inexistência de cuidador foi a principal causa para a entrada em ERPI. A informação sobre a indisponibilidade, a fragilidade ou a ausência total de cuidador informal foi analisada em $73 \%$ dos estudos. Em resumo, os estudos sugerem-nos três âmbitos diferentes apresentando um teor causal entre a rede de apoio informal e o ingresso de pessoas idosas em ERPI (Cf. Tabela 3): 
Tabela 3. Resultados sintetizados relacionados com o preditor de evidência forte "Ausência de cuidador: indisponibilidade de familiares para cuidar, fragilidade ou ausência de rede de apoio"

\begin{tabular}{ll}
\hline \multicolumn{1}{c}{ Âmbito principal } & \multicolumn{1}{c}{ Âmbito secundário } \\
\hline & - Viver sozinho/a; \\
Inexistência de cuidador informal & - Não ter filhos; \\
e/ou inexistência de rede de & - Ter poucos filhos; \\
suporte familiar. & - Ocorrência de viuvez; \\
& - Opção de se manter solteiro/a; \\
& - Separação/divórcio. \\
& - Incapacidade de o cônjuge cuidar; \\
& - Stress do cuidador; \\
Incapacidade do cuidador principal & - Sobrecarga do cuidador, \\
em cuidar. & - Frustração do cuidador informal em não conseguir \\
& responder às necessidades daquele que é cuidado. \\
& - Resfriamento de vínculos afetivos; \\
Ausência de disponibilidade da & - Conflitos familiares e exclusão familiar; \\
rede familiar para cuidar. & - Problemas de saúde do cuidador; \\
& - Abandono. \\
\hline Fonte: Elaboração própria.
\end{tabular}

Fonte: Elaboração própria.

Os dados apresentados na Tabela 3 são particularmente reveladores da multiplicidade de causas que sustentam a variável relacionada com o contexto sociofamiliar como um dos principais preditores da institucionalização de pessoas idosas em contextos de ERPI.

Primeiramente analisamos os dados que provam a existência cada vez maior de pessoas idosas a viver sozinhas no domicílio ou exclusivamente com outras pessoas com 65 ou mais anos. Entre 2001 e 2011 , segundo os dados mais recentes publicados relacionados com esta matéria e a que tivemos acesso oriundos do Instituto Nacional de Estatística (2012), em Portugal, passou-se de 942.594 para 1.119.324 as pessoas que viviam sozinhas, correspondendo a um aumento de $27 \%$. Em termos sociodemográficos o perfil de pessoas que vivem sozinhas, segundo a mesma fonte, tende a ser: (a) pessoas que vivem no interior do país; (b) mulheres; (c) viúvas; (d) com níveis baixos de escolaridade; e (e) em situação de reforma (e.g., Moreira, 2020). Acresce a este perfil, o facto de tratarmos de pessoas com (a) rendimentos muito baixos; (b) com redes informais frágeis (ou porque os familiares estão longe e/ou porque os vizinhos estão também eles nas mesmas condições); (c) suporte intergeracional tendencialmente inexistente; (e) problemas de isolamento; e (f) progressiva incapacidade (e.g., Moreira, 2020).

Assim, a este nível, algumas das razões que se apontam para a institucionalização parecem indicarnos: (a) a pessoa idosa se sentir sozinha ou (b) por decisão da família (e.g., por dificuldade ou distância da pessoa idosa, residir no domicílio sozinha, ter alguma doença tendencialmente incapacitante), que considera que o seu familiar terá melhor apoio em contexto institucional (Moreira, 2020).

Um segundo aspeto prende-se com a incapacidade do cuidador principal em cuidar ou a ausência de disponibilidade da rede familiar para cuidar. A este propósito, é conveniente recordar que em Portugal, existem 827.000 cuidadores e cuidadoras informais, ou seja, cerca de $8 \%$ da população, $25 \%$ dos quais (aproximadamente 200.000) a tempo inteiro (Eurocarers, 2019), sendo estes essencialmente mulheres (Alves et al., 2020; Moreira, 2020; Observatório Português dos Sistemas de Saúde [OPSS], 2015). Para além destes dados, destacamos o facto de existirem cerca de 110.355 pessoas dependentes das AVD e AIVD, das quais 48.454 estariam acamadas (Observatório Português dos Sistemas de Saúde [OPSS], 2015).

Centrando-nos no estudo de Alves e colaboradores (2020), averigua-se que os cuidadores eram (a) essencialmente mulheres; (b) com média de idade de 60,6 anos; (c) aposentados e com baixas reformas; (d) eram casados ou filhos da pessoa que era cuidada; (e) prestavam cuidados contínuos; (f) com baixo nível educacional (i.e., até ao $4^{\circ}$ ano de escolaridade); e (g) enfrentavam problemas médicos que envolviam maiores gastos com saúde (e.g., medicamentos, ajudas de custo, consultas médicas). No que diz respeito aos destinatários dos cuidados: (a) a média de idade era de 88,7 anos; (b) era viúvo, solteiro ou divorciado; e (c) eram muito dependentes e com comprometimento cognitivo, dados que encontram paralelo com o estudo de Brandão et al. (2017).

Não obstante, é preciso referir que o processo natural de envelhecimento faz-se acompanhar, em regra, de um período de fragilidade decorrente da existência de doenças crónicas e/ou episódios 
extemporâneos levando a maior dependência de terceiros, transferindo para o espaço doméstico maior pressão naquilo que tem de ver com o ato cuidativo e/ou a procura de cuidados formais. Neste sentido, a solidariedade familiar que pretenda garantir um cuidado ótimo requer não apenas disponibilidade de tempo, de recursos económicos, de infraestruturas domiciliares adaptadas, de apoios estatais e institucionais complementares (e.g., Serviço de Apoio Domiciliário), de regulamentação laboral (e.g., flexibilização ou redução de horário laboral, gozo de licenças, entre outros), mas também de reconhecimento (e.g., social), conhecimento e habilidades técnicas básicas de saúde (Alves et al., 2020; Pimentel, 2012). Nesta medida, a necessidade de cuidados formais, tal como comprovámos na Tabela 3, tem grande impacto na institucionalização da pessoa idosa, desde logo porque, como comprovou Alves et al. (2020), existe pelo menos quatro necessidades não atendidas pelos cuidadores informais: (a) a necessidade de apoio financeiro; (b) a necessidade de apoio do cuidador corresponde ao nível do cuidador familiar/informal; (c) a necessidade de atenção primária/especialidades médicas; e (d) a necessidade de regulação do trabalho corresponde ao nível do modelo das políticas nacionais.

A este nível, de entre os estudos analisados evidenciamos, o desgaste e a sobrecarga dos cuidadores. Dados indicam que cuidar de pessoas idosas com grandes necessidades resulta numa carga considerável sobre os cuidadores informais, podendo, entre outros, causar sentimentos de angústia e stress ou a perceção de que as demandas do cuidado excedem as capacidades e recursos disponíveis (e.g., Alves et al., 2020; Betini et al., 2017). Diversas especificidades agravam essa sobrecarga, concretamente, a idade avançada dos próprios cuidadores, o seu estado geral de saúde e a extensão reduzida da rede de apoio (Brandão et al., 2017). A título de exemplo, no World Alzheimer Report 2019 (Alzheimer's Disease International [ADI], 2019) foi identificado que mais de $50 \%$ dos cuidadores de pessoas com demência viam a sua saúde prejudicada devido às responsabilidades inerente ao ato de cuidar. No mesmo relatório é referido ainda que mais de $60 \%$ dos cuidadores de pessoas com demência encontram dificuldades na manutenção da vida social em resultado das responsabilidades do cuidado.

Neste sentido, na pesquisa de Betini et al. (2017) é referido que a tensão e sentimentos negativos associado ao ato cuidativo tendem a afetar a qualidade de vida do cuidador e capacidade para continuar as atividades de cuidado, levando, não poucas vezes, à institucionalização do destinatário do cuidado. Nesta linha, apresentam-se como algumas das principais razões de institucionalização em ERPI baseadas na variável sociofamiliar: (a) a insuficiente habilidade do cuidador; (b) os problemas de saúde do cuidador informal; (c) o comportamento difícil da pessoa idosa que é cuidada; e (d) o aumento da dependência daquele que é cuidado.

Na substância, a elevada dependência das pessoas que beneficiem do ato cuidativo, bem como a fragilidade daqueles que cuidam, sugere a institucionalização em ERPI como principal solução ao alívio de stress/pressão experienciados pelos cuidadores informais, assim como a prestação de cuidados de cuidadores formados e com experiência no ato cuidativo às pessoas idosas.

\section{Discussão}

O presente estudo evidenciou que a ausência de cuidador informal decorrente da indisponibilidade de familiares para cuidar e da fragilidade ou ausência de rede de apoio, a existência de problemas psicopatológicos, neuropsiquiátricos e neuropsicológicos em pessoas idosas e a dependência de cuidados nas AVD e AIVD correspondem aos principais preditores da institucionalização de pessoas idosas em contextos de ERPI.

A ocorrência de problemas psicopatológicos, neuropsiquiátricos e neuropsicológicos nas pessoas mais velhas, aumentam a probabilidade de necessidade de apoio de cuidadores, sendo a manutenção do bem-estar assegurado, numa primeira fase, pela família. No entanto, os dados mais recentes publicados e a que tivemos acesso (Instituto Nacional de Estatística [INE], 2012) não seguem a mesma linha, isto é, em Portugal existe um elevado número de pessoas idosas que vivem sozinhas. Contrariamente a este último aspeto, quando a solidariedade familiar providencia o cuidado de pessoas idosas com doenças do tipo diagnóstico de Alzheimer ou demência, conforme apurámos neste trabalho, experiencia-se elevado desgaste e sobrecarga, uma vez que as AVD e as AIVD da pessoa que é cuidada ficam comprometidas, exigindo maior responsabilidade imputada aos cuidadores levando a maiores níveis de stress. Qualquer dos quadros 
apresentados, sugere-nos como uma potencial causa que pode determinar a institucionalização de pessoas idosas em ERPI.

Carece de se atender que, o papel da sociedade aliado à ocorrência de demência e/ou diagnóstico de Alzheimer devem ser enfatizados, na verdadeira medida em que o número de pessoas idosas que vivem sozinhas e o número de pessoas idosas com demência e/ou diagnóstico de Alzheimer e/ou com restrições à funcionalidade deverá aumentar substancialmente nas próximas décadas (Lippi Bruni e Ugolini, 2016). Durante o mesmo período, a disponibilidade de cuidadores informais provavelmente diminuirá (Pimentel, 2012) devido à implosão demográfica e às transformações ocorridas na composição e dimensão da família, à redução da taxa de natalidade e à participação da mulher no mercado de trabalho (Moreira, 2020; Pimentel, 2012). Em suma, é altamente provável que a demanda pela institucionalização entre as pessoas idosas aumente drasticamente nos próximos anos (e.g., Hajek et al., 2015).

Os resultados obtidos oferecem aos múltiplos profissionais que trabalham com pessoas idosas, por um lado alertas acerca dos públicos que atualmente recorrem às ERPI e aqueles que futuramente se socorrerão de cuidados formais e por outro lado indicadores valiosos a fim de oferecer cuidados adequados às pessoas idosas no futuro. Este facto pode, através de um maior conhecimento sobre os indicadores da institucionalização, direcionar medidas para melhorar as estratégias de acolhimento e garantia de bem-estar dessa população. Simultaneamente, este estudo também disponibiliza informações importantes às famílias por forma a que estes atentem às possibilidades de prevenção e consigam identificar quando a institucionalização é, de facto, indicada.

Destaca-se a relevância que estudos como este, que investigam as causas da institucionalização, têm para os profissionais do social, neste caso os Assistentes Sociais, que por estarem bastante próximos das pessoas idosas e das famílias no domicílio (i.e., quando o Serviço de Apoio Domiciliário é uma alternativa viável) e em ERPI, têm como potencialidade coordenar e efetivar o cuidado com qualidade, bem como a garantia da dignidade, do respeito, da justiça e dos direitos humanos.

Ao longo dos tempos assistiu-se à reconfiguração do agir dos Assistentes Sociais, muito por força dos estados democráticos, pelo conhecimento das ciências sociais e pelo desenvolvimento e integração dos princípios fundamentais dos Direitos Humanos nos processos societários, substituindo a ação curativa e paliativa por uma intervenção inovadora assente na anticaridade e antifilantropia (Carvalho, 2014).

No entanto, mesmo antes de a velhice se tornar um desafio para as sociedades, as pessoas mais velhas já representavam um "objeto" de intervenção do Serviço Social, ainda que se tratasse de uma intervenção curativa (Mouro, 2013).

No caso português, a queda do regime ditatorial e o estabelecimento de um sistema democrático (Carvalho, 2014), em simultâneo com o aumento do número de pessoas idosas e a recomposição da morfologia dos agregados familiares, permitiu desocultar a velhice tornando-a um fenómeno visível. Isto é, a velhice passa a ser vista como uma questão de direitos humanos e sociais. Na substância, a intervenção com as pessoas idosas que tendia a ser um exclusivo da esfera privada e familiar (Guedes, 2014), passa a ser uma problemática a incluir na esfera coletiva e política (Carvalho, 2014). Esta problemática é o ponto de partida para a reflexão de Joana Guedes (2014) e de Helena Mouro (2013) ao advogarem que a velhice passa a ser reconhecida como um problema social a partir do momento em que na história se inicia a tomada de medidas de resposta aos desafios a ela associados, com fim último à sua resolução ou mitigação.

Não obstante, o reconhecimento da velhice como um desafio que progressivamente transita do espaço privado para a esfera coletiva, faz aumentar a legitimidade do fenómeno, materializando-se através dos sistemas de proteção e apoio social. Destes sistemas de apoio emergem normativos regulatórios, (re)distributivos e constitutivos, bem como uma vasta oferta de equipamentos da rede solidária e do setor privado dedicados à prestação de cuidados formais às pessoas idosas. Com estas transformações micro, macro e meso, tornam-se não apenas fundamentais como também em número avultado (Gabinete de Estratégia e Planeamento [GEP], 2021) as ERPI enquanto equipamentos residenciais que, dependendo das circunstâncias, atuam em paralelo com demais sistemas sociais e/ou em exclusivo no ato cuidativo às pessoas idosas.

Os dados trabalhados neste estudo, sugerem-nos alertas acerca dos desafios vários imputados às ERPI e, por conseguinte, aos profissionais. Neste seguimento, é da mais elementar pertinência que os profissionais que intervêm nas ERPI, onde historicamente se incluem Assistentes Sociais, constituam movimentos críticos individuais e coletivos de reconceptualização da atuação. Isto é, o facto de se tratarem de beneficiários do cuidado muito idosos que apresentam necessidades múltiplas, por exemplo, emocionais, 
físicas/motoras e sociais (Gabinete de Estratégia e Planeamento [GEP], 2019, 2021), obriga os profissionais, por exemplo a desenvolverem ações centradas na pessoa e menos na tarefa. Assim, se por um lado nestes estabelecimentos residenciais se assiste timidamente a alterações vanguardistas na forma como se cuida, também é verdade que ainda permanecem constrangimentos vários (e.g., cuidados pouco flexíveis baseados em lógicas curativas assentes na proteção e segurança e não na autonomia e liberdade) inviabilizadores do garante da participação ativa e da cidadania das pessoas mais velhas.

A incapacidade de diligenciar pontos de viragem na gestão do ato cuidativo formal de pessoas idosas adensa a rotinização daquele que deveria ser um ato essencialmente relacional e humanizado. Apostar em abordagens integradas, prospetivas e sustentáveis suscitadas pela conversão dos problemas abstratos derivados da intervenção social em operações científicas práticas pode levar, quando a nós, à alteração de paradigmas de atuação dos Assistentes Sociais. Lógicas de aliança entre a pesquisa académico-científica e o conhecimento advindo dos serviços ou associada ao trabalho profissional configuram-se em dinâmicas transformadoras da realidade social (Moraes, 2017).

No dizer de Carlos António de Souza Moraes (2017), os Assistentes Sociais para além de serem fundamentais no trabalho de campo, são igualmente preponderantes na produção de conhecimento em matérias e problemáticas intimamente ligadas à sua prática profissional. Esse exercício, eminentemente reflexivo, baseado no conhecimento-processo e na atuação e pesquisa em rede pode levar i) a desvendar questões enigmáticas do trabalho profissional e ii) indicar novos caminhos à pesquisa científica.

Na substância, o que devemos reter daqui é o facto de o Serviço Social conter na sua matriz a perspetiva de garante dos Direitos Humanos, da dignidade humana e da justiça social. Como tal, e em nome de uma sociedade modernizada composta por sistemas familiares que cada vez mais se socorrem da ajuda e apoio institucional de equipamentos da rede solidária e do setor privado, é imperativo rever e otimizar muitos princípios que constituem pilares de admissão das pessoas idosas em ERPI. A admissibilidade em ERPI sem critérios, a ausência de respostas alternativas às estruturas residenciais e a sectorização agigantam fossos individuais e coletivos e perigam o equilíbrio social futuro.

\section{Conclusão}

Embora escasseie evidência científica que permita relacionar a experienciação da velhice e aquilo que leva as pessoas mais velhas a ingressar em ERPI, os resultados deste trabalho confirmam a vulnerabilidade desta população, verificando-se que as pessoas idosas que vivem em contextos residenciais se socorrem destes serviços fundamentalmente porque experienciam a perda de saúde e da autonomia a propósito, entre outros, da existência de problemas psicopatológicos, neuropsiquiátricos e neuropsicológicos. Por outro lado, o facto de a rede de suporte familiar e/ou cuidador informal não existir ou não ter disponibilidade para cuidar e o cuidador principal ser incapaz de garantir o ato cuidativo (por ausência de competências e sobrecarga) sugere maior probabilidade de as pessoas idosas ingressarem em ERPI.

Para além disso é conhecida a tendência que as pessoas idosas que vivem em estruturas residenciais têm para apresentar baixos níveis de participação, de capacidade de tomada de decisão, de autoconceito e de autoestima, que podem ter consequências negativas, como por exemplo a apatia e a passividade, se não forem consideradas atempada e adequadamente. Dada a importância da avaliação diagnóstica na prática gerontoinstitucional, os profissionais do social e da saúde das ERPI, onde se incluem os Assistentes Sociais, beneficiam de competências capazes de atenuar os efeitos das perdas sociais e naturais do processo de senescência do ser humano que, conforme comprovámos, se assiste no ingresso em contextos institucionais. Este desígnio implica ter atenção especial ao potencial, às vontades, às competências e aos talentos preservados das pessoas idosas, garantindo, por um lado o conteúdo ético, político e axiológico norteador das ERPI, e por outro lado o bem-estar e a felicidade das pessoas mais velhas.

Espera-se que o presente estudo sirva como base teórico-científica para outras pesquisas no âmbito do Serviço Social e outras ciências visando o embasamento e aprofundamento científico para realizar ações sociais mais efetivas, como por exemplo, junto daqueles que estão em alto risco de institucionalização poderem ser educados para preparar o seu futuro (e.g., Estatuto do Maior Acompanhado), e antecipando quem os ajudará a tomar decisões e/ou quem decidirá por si. Poder-se-á, assim, perpetuar e renovar o ato cuidativo junto das pessoas idosas, por forma a garantir uma velhice mais próspera e digna. 
Por fim, os preditores que conduzem as pessoas idosas a viverem numa ERPI e as múltiplas variáveis que estão na sua base revelam ainda a necessidade emergente de investigações mais aprofundadas e multidisciplinares, nomeadamente de natureza qualitativa, nas quais o Serviço Social deve ser incluído.

\section{Referências bibliográficas}

Alves, S., Ribeiro, O. e Paúl, C. (2020): "Unmet needs of informal carers of the oldest old in Portugal", Health and Social Care in the Community, 28 (6): 2408-2417. https://doi.org/10.1111/hsc.13063

Alzheimer's Disease International [ADI] (2019): World Alzheimer Report 2019. Attitudes to dementia. Disponível na web: https://www.alzint.org/resource/world-alzheimer-report-2019/

Alzheimer Europe (2019): Dementia in Europe Yearbook 2019 Estimating the prevalence of dementia in Europe. Disponível na web: https://www.alzheimer-europe.org/Publications/Dementia-in-Europe-Yearbooks

Betini, R. S., Hirdes, J. P., Lero, D. S., Cadell, S., Poss, J. e Heckman, G. (2017): "A longitudinal study looking at and beyond care recipient health as a predictor of long-term care home admission", BMC Health Services Research, 17 (1): 1-10. https://doi.org/10.1186/s12913-017-2671-8

Brandão, D., Ribeiro, O., Oliveira, M. e Paúl, C. (2017): "Caring for a centenarian parent: an exploratory study on role strains and psychological distress", Scandinavian Journal of Caring Sciences, 31 (4): 984-994. https://doi.org/10.1111/scs.12423

Cabral, M. V. (2017): "O envelhecimento sociodemográfico e os seus riscos", em Ferreira, P. M.; Cabral, M. V. e Moreira A. eds.: Envelhecimento na Sociedade Portuguesa. Pensões, Família e Cuidados: 99-109. Lisboa: Imprensa de Ciências Sociais.

Carvalho, M. I. (2014): "Serviço Social e intervenção com idosos: Desafios atuais", em Carvalho, M. I. e Pinto, C. eds.: Serviço Social. Teorias e Práticas. 421-436. Lisboa: PACTOR.

Comissão Europeia [CE] (2021): Livro verde sobre o envelhecimento. Promover a responsabilidade e a solidariedade entre gerações. Comissão Europeia. Disponível na web: https://bit.ly/3mGzcVO

De Medeiros, M. M., Carletti, T. M., Magno, M. B., Maia, L. C., Cavalcanti, Y. W. e Rodrigues-Garcia, R. C. (2020): "Does the institutionalization influence elderly's quality of life? A systematic review and meta-analysis", BMC Geriatrics, 20 (1): 1-25. https://doi.org/10.1186/s12877-020-1452-0

Eurocarers (2019): Number of carers and existing support measures across the EU. Disponível na web: https://bit.ly/3il2zpt

Ferreira da Silva, D., Talitha Fernandes Barbosa, K., Marilaid da Silva Honório, G., Texeira de Carvalho Dias, C., Pessoa da Rocha Leal, N. e Maria Rodrigues Lopes de Oliveira, F. (2020): "Institucionalização da pessoa idosa: determinantes e caracterização sociodemográfica", Cultura de Los Cuidados. Revista de Enfermeria y Humanidades, 24 (58): 217-228. https://doi.org/10.14198/cuid.2020.58.19

Gabinete de Planeamento e Estratégia [GPE] (2021): Carta Social-Rede de Serviços e Equipamentos 2019Relatório 2019. Disponível na web: http://www.cartasocial.pt/pdf/csocial2019.pdf

- (2019): Carta Social-Rede de serviços e equipamentos-Relatório 2018. Disponívem na web: http://www.cartasocial.pt/pdf/csocial2018.pdf

Goodwin, J. S., Howrey, B., Zhang, D. D. e Kuo, Y. F. (2011): "Risk of continued institutionalization after hospitalization in older adults", Journals of Gerontology-Series A Biological Sciences and Medical Sciences, 66 A (12): 1321-1327. https://doi.org/10.1093/gerona/glr171

Guedes, J. (2014): "Cuidados formais a idosos. Desafios inerentes à sua participação", em Fonseca, A. M. ed.: Envelhecimento, saúde e doença. Novos desafios para a prestação de cuidados a idosos, 184-209. Lisboa: Coisas de Ler.

Hajek, A., Brettschneider, C., Lange, C., Posselt, T., Wiese, B., Steinmann, S., Weyerer, S., Werle, J., Pentzek, M., Fuchs, A., Stein, J., Luck, T., Bickel, H., Mösch, E., Wagner, M., Jessen, F., Maier, W., Scherer, M., RiedelHeller, S. G., e König, H. H. (2015): "Longitudinal predictors of institutionalization in old age", PLoS ONE, 10 (12), e0144203. https://doi.org/10.1371/journal.pone.0144203

Instituto Nacional de Estatística [INE] (2020): Tábuas de Mortalidade em Portugal. Disponível na web: https://bit.ly/3sZgdYe

- (2012): Mais de um milhão e duzentos mil idosos vivem sós ou em companhia de outros idosos. Disponívem na web: https://bit.ly/3rQpEYS

Lippi Bruni, M. e Ugolini, C. (2016): "Delegating home care for the elderly to external caregivers? An empirical study on Italian data", Review of Economics of the Household, 14 (1): 155-183. https://doi.org/10.1007/s11150-014-9253-x 
Lopes, V. M., Scofield, A. M. dos S., Alcântara, R. K. de, Fernandes, B. Karen C., Leite, S. F. e Borges, C. L. (2018): "O que levou os idosos à institucionalização?", Revista de Enfermagem UFPE on Line, 12 (9): 2428-2435. https://doi.org/10.5205/1981-8963-v12i9a234624p2428-2435-2018

Luppa, M., Luck, T., Weyerer, S., König, H. H., Brähler, E. e Riedel-Heller, S. G. (2009): "Prediction of institutionalization in the elderly. A systematic review", Age and Ageing, 39 (1): 31-38. https://doi.org/10.1093/ageing/afp202

Mendes, M. F. (2017): "Envelhecimento e fecundidade: uma antevisão do nosso futuro demográfico", em Cabral, M. V., Ferreira, P. M. e Moreira A. eds.: Envelhecimento na sociedade portuguesa. Pensões, família e cuidados: 111-140. Lisboa: Instituto de Ciências Sociais.

Moraes, C. A. de S. (2017): "Pesquisa em Serviço Social: concepções e críticas", Revista Katálysis, 20 (3): $390-$ 399. https://doi.org/10.1590/1982-02592017v20n3p390

Moreira, M. J. (2020): Como envelhecem os portugueses. Envelhecimento, Saúde, Idadismo. Lisboa: Fundação Francisco Manuel dos Santos.

Mouro, H. (2013): "Envelhecimento, politicas de intervenção e Serviço Social", em Carvalho, M. I. de ed.: Serviço Social no envelhecimento: 17-34. Lisboa: PACTOR.

Nogueira, M. F., Lima, A. A., Trigueiro, J. V., Torquato, I. M., Henriques, M. E. de M. e Alves, M. do S. C. (2016): "Comparando a qualidade de vida de idosos institucionalizados e não-institucionalizados", Revista Enfermagem, 24 (5). https://doi.org/10.12957/reuerj.2016.28185

Observatório Português dos Sistemas de Saúde [OPSS] (2015). Acesso aos cuidados de saúde. Um direito em risco? Relatório de Primavera 2015. Disponivel na web: https://bit.ly/3mJQaTb

Organisation for Economic Co-operation and Development [OECD] (2019): Health at a Glance 2019. OECD Indicators. Disponível na web: https://doi.org/https://doi.org/10.1787/4dd50c09-en

Pimentel, L. G. (2012): A prestação de cuidados a pessoas idosas dependentes: uma análise das relações familiares intergeracionais e de germanidade. Instituto Universitário de Lisboa: Superior de Ciências do Trabalho e da Empresa. (Tese de PhD).

Pimentel, L. G. e Albuquerque, C. P. (2010): "Solidariedades Familiares e o Apoio a Idosos. Limites e Implicações", Textos \& Contextos, 9 (2): 25-263. https://bit.ly/3FtPxps

Pinto, D. C. (2013): Por que vão os idosos para lares? Determinantes no internamento de pessoas maiores de 65 anos em instituições de longa permanencia. Universidade de Lisboa: Instituto Superior de Ciências Sociais e Políticas. (Dissertação de mestrado).

Pinzón-Pulido, S., Garrido Peña, F., Reyes Alcázar, V., Lima-Rodríguez, J. S., Raposo Triano, M. F., Martínez Domene, M. e Alonso Trujillo, F. (2016): "Factores predictores de la institucionalización de personas mayores en situación de dependencia en Andalucía", Enfermeria Clinica, 26 (1): 23-30. https://doi.org/10.1016/j.enfcli.2015.08.003

PORDATA (2021): População residente: total e por grandes grupos etários. Disponível na web: https://bit.ly/3xaKVzx

— (2021a): Esperança de vida à nascença: total e por sexo (base: triénio a partir de 2001). Disponível na web: https://bit.ly/3BujeUW

- (2021b): Esperança de vida aos 65 anos: total e por sexo (base: triénio a partir de 2001). Disponível na web: https://bit.ly/3uYrjyN

- (2021c): Em 2019 continuou a tendência de redução do risco de pobreza. Em 2020 assistiu-se a uma redução da privação material e ao agravamento das condições de saúde-2020. Disponível na web: https://bit.ly/3mHwoYH

- (2020): Indicadores fundamentais de saúde apontam para melhoria nos anos recentes, embora alguns mantenham níveis inferiores aos médios da União Europeia (UE-28)-2008-2019. Disponível na web: https://bit.ly/3iMpQ9P

- (2019): População residente: total e por grandes grupos etários. Quantos são os jovens, os idosos ou as pessoas em idade activa? Disponível na web: https://bit.ly/3w7Xknl

Rodrigues, R. (2017): "Cuidados de longa duração para idosos no contexto europeu: múltiplas soluções para um problema comum?", em Ferreira, P. M.; Moreira, M. V. e Cabral, A. eds.: Envelhecimento na sociedade portuguesa. Pensões, família e cuidados. 165-179. Lisboa: Imprensa de Ciências Sociais.

Rosa, M. J. (2020): Um tempo sem idades (The age of no age). Ensaio sobre o envelhecimento da população. Lisboa: Tinta da China.

Serviço Nacional de Saúde [SNS]. (2020): Estruturas Residenciais para idosos. Portugal regista evolução positiva da Covid-19 nos lares. Disponívem na web: https://bit.ly/3AKfQEj 


\section{Breve CV do autor:}

Ricardo Crispim é Doutorando no Programa Interuniversitário de Doutoramento em Serviço Social na FPCEUC. Mestre em Serviço Social pela mesma instituição e mestre em Ciências da Educação pela ESECS-IPLeiria. Duplamente licenciado em Educação Social e em Serviço Social pela ESECS-IPLeiria. Interventor Social no campo da Gerontologia em ERPI. As principais linhas de pesquisa são: Intervenção Social, Políticas Sociais, Envelhecimento Ativo e Saudável, Participação. 